\title{
Analytic Dependence of Orthogonal Polynomials
}

Enrico Laeng

\section{Introduction}

One way we can define and construct orthogonal polynomials is by applying the Gram-Schmidt orthogonalization process to the sequence of powers $1, x, x^{2}, \ldots$ More precisely, we embed these power-functions in $L^{2}(d \mu)$, where $d \mu$ is a probability measure living on some space (usually the closed interval $[-1,1]$, the real line $\mathbf{R}$, or the unit circle $\mathbf{T}$ ), and then we orthogonalize with respect to the scalar product induced by the measure $d \mu$. In the classical theory, $d \mu$ is usually absolutely continuous with respect to the Lebesgue Measure, and given by some very explicit weight function, but recently, in $\mid \mathrm{CM}]$, R. R. Coifman and M. Murray have proposed a different analytic approach to the study of general orthogonal polynomials, based on a «perturbation theory» of the orthogonalization process.

The approach of R. R. Coifman and M. Murray allows one to study orthogonal polynomials within the framework of non-linear Fourier Analysis. As in similar studies of other non-linear problems (dependence of the Riemann mapping on the domain, shape of water waves, Navier-Stokes equation) the main result consists in showing that some relevant operator quantities are analytically dependent on a B.M.O. parameter. In this kind of analysis, the theory of weighted norm inequalities, $A_{p}$ weights, and the relationships between good weights and BMO, play a central role (an excellent source of reference for this is [GCRF]).

This particular problem belongs also to the context of Toda flows (see [DLT]) where a certain non-linear matrix differential equation admits closed form solutions. These solutions are a «flow» of infinite tridiagonal matrices that 
can be interpreted as the three-term recursion relation attached to the orthogonal polynomials.

In $[\mathrm{CM}]$ the program is carried out in detail starting from two situations: polynomials on the circle $\mathbf{T}$ orthogonal with respect to Lebesgue measure, and polynomials on $\mid-1,1]$ orthogonal with respect to a Jacobi weight. In this work we carry out the same program starting from a set of polynomials on $\mathbf{T}$ first introduced by Szegö, in fact used by him as a tool for the proof of his equiconvergence theorems.

We then show some new results. Using the closed subspace VMO, we give a better characterization of the BMO region where the analyticity result holds. This can be seen as the definition of a very general set of polynomials that can be "analytically perturbed». The relationship with the theory of Toda flows is exploited to derive new identities that can be used interchangeably with the Kerzman-Stein formula, shedding a new light on the connections among the different operators involved. The flow itself is expressed in terms of conjugation by a self-adjoint projection $Q$ whose dependence from the parameter gives rise to interesting formulae. In fact, starting from Chebychev polynomials, the first Gateaux differential of $Q$ can be expressed via Hilbert transforms and a bilinear singular integral operator first considered by Calderón (see Section 7). The $L^{2}$-boundedness of this operator is still an open problem.

We do not deal with numerical applications, but let us note that this theory can provide some useful computer tools. The Kerzman-Stein formula can be used to efficiently compute orthogonal polynomials relative to standard and non-standard weights. The analyticity results suggest the possibility of building fast algorithms (complexity $n \log n$ ) to convert an orthogonal expansion relative to a fixed set of polynomials into the expansion relative to another set of polynomials, provided they are within the "analyticity range» (see Theorem 1 in Section 6, and se also $[\mathrm{AR}]$ for an example of numerical conversion from Chebychev polynomials expansions into Legendre polynomials expansions).

\section{Orthogonal Polynomials Dependent on a BMO Parameter}

Consider a «perturbed» space $L^{2}\left(u^{2} d \mu\right)$ where $u(x)=e^{b(x)}$ is a suitable function. Notice that if $b(x)$ is close to zero (in some Banach Space norm which will be chosen later), then the new measure

$$
u^{2}(x) d \mu(x)=e^{2 b(x)} d \mu(x)
$$

is «close» to the original one $d \mu$. 
Now, given $f \in L^{2}\left(u^{2} d \mu\right)$, we can develop it into a series of orthogonal polynomials (the polynomials which are orthogonal with respect to $u^{2} d \mu$ ) and consider the partial sums $S_{n} f$ of this series. Notice that, for a fixed measure $u^{2} d \mu$ living on a fixed space, the orthogonal polynomials are uniquely determined (up to a normalization), and so is $S_{n}$. Notice also that the partial sums operator $S_{n}$ can be thought of as an operator-valued function of $b$. In fact, we want to study the mapping

$$
b \rightarrow S_{n}(b) \text {. }
$$

It is hard to study (2) directly because $S_{n}(b)$, in spite of being a well-defined projection from $L^{2}\left(u^{2} d \mu\right)$ into polynomials of degree equal or less than $n$, acts on different $L^{2}$ spaces as $b$ varies. On the other hand, we can look at

$$
b \rightarrow V_{n}(b)
$$

where

$$
V_{n}(b)=u S_{n}(b) u^{-1}=e^{b} S_{n}(b) e^{-b}
$$

With a little abuse of notation, in the above definition we use a function to denote the operator of multiplication by that function. It is easy to check that $V_{n}(b)$ always acts on $L^{2}(d \mu)$, that the $L^{2}(d \mu)$-boundedness of $V_{n}(b)$ is equivalent to the $L^{2}\left(u^{2} d \mu\right)$-boundedness of $S_{n}(b)$, and that the two operator norms are equal.

The mapping (3) can now be seen in the context of calculus on Banach Spaces, see for example $[B]$, where notions like continuity, differentiability, and analyticity and well defined.

We claim that the «natural» Banach Space for $b$ is BMO (Bounded Mean Oscillation) and that in «many» situations, depending on the choice of the measure space we start with, the dependence (3) is in fact analytic. We will make this claim precise later, and we will prove it.

Let us notice at this point that a further simplification in the study of this problem is brought about by a remarkable formula due to Kerzman and Stein $[\mathrm{KS}]$. The formula is

$$
V=P\left(I+\left(P-P^{*}\right)\right)^{-1}
$$

where $V$ is a self-adjoint projection sending a Hilbert Space $H$ into a proper closed subspace $K$ and $P$ is an oblique projection (non self-adjoint) also sending $H$ into $K$.

The first step in the proof of (5) is to show that the operator $I+\left(P-P^{*}\right)$ 
is invertible, but in fact, since $\left(P-P^{*}\right)^{*}=P^{*}-P=-\left(P-P^{*}\right)$, we see that $P-P^{*}$ is skew adjoint, which means that its spectrum is purely imaginary. In particular $-1 \notin \operatorname{spec}\left(P-P^{*}\right)$, which shows the invertibility of $I+\left(P-P^{*}\right)$. We can now rewrite (5) as

$$
V\left(I+\left(P-P^{*}\right)\right)=P
$$

and this is the same as

$$
P=V+V P-V P^{*}=V+P-V P^{*},
$$

which simplifies into

$$
V P^{*}=V
$$

Finally, we prove this last operator equality using an elementary lemma from Functional Analysis (see for example $[R \mid$ p. 296) and the following chain of scalar-product equalities, which hold for any element $h \in H$ :

$$
\left(V P^{*} h, h\right)=\left(P^{*} h, V h\right)=(h, P V h)=(h, V h)=(V h, h) .
$$

Having proven (5), we now apply it to our situation by choosing

$$
V=V_{n}(b)=e^{b} S_{n}(b) e^{-b}
$$

and

$$
P=P_{n}(b)=e^{b} S_{n}(0) e^{-b} .
$$

Notice that (with the same abuse of notation) we have used $e^{b}$ to indicate the operator of multiplication by $e^{b}$. Both $V_{n}(b)$ and $P_{n}(b)$ are projection operators from $L^{2}(d \mu)$ into the same closed subspace; $V_{n}(b)$ is self-adjoint while $P_{n}(b)$ is an oblique projection that satisfies

$$
P_{n}(b)^{*}=P_{n}(-b)
$$

The Kerzman-Stein formula (5) tells us that the analytic properties of $P_{n}(b)$ as an operator-valued function of $b$ are inherited by $V_{n}(b)$. This is a great simplification because it is easier to deal explicitly with the integral kernel of $S_{n}(0)$ than that of $S_{n}(b)$. So, once we have proven the uniform analycity of $P_{n}(b)$, we have proven it also for $V_{n}(b)$. To be self-contained, let us state here the following

Definition 1 of Uniform Analyticity for a sequence $\left\{P_{n}(b)\right\}$ of operatorvalued functions of $b \in B$ (some Banach Space). 
The sequence $\left\{P_{n}(b)\right\}$ is uniformly (real) analytic in a neighborhood of $0 \in B$ if and only if there exists $\delta>0$ such that for every $f \in L^{2}(d \mu)$ and all $b$ with $\|b\|_{B}<\delta$ we have

$$
P_{n}(b) f=\sum_{k=0}^{\infty} \Lambda_{n k}(b, \ldots, b, f) \quad \text { for every } n
$$

where $\Lambda_{n k}$ is a bounded $(k+1)$-multilinear operator sending $B^{k} \times L^{2}(d \mu)$ into $L^{2}(d \mu)$ and satisfying the estimate

$$
\left\|\Lambda_{n k}(b, \ldots, b, f)\right\|_{L^{2}(d \mu)} \leq c^{k+1}\|b\|_{B}^{k}\|f\|_{L^{2}(d \mu)} .
$$

The constant $c$ in (9) does not depend on $n$. Notice that $\Lambda_{n k}(b, \ldots, b, \bullet)$ is the $k^{\text {th }}$ Gateaux differential of $P_{n}(b)$ at 0 in the direction of $b$.

A similar definition can be given, when $b$ belongs to a complex Banach Space, for the uniform holomorphy of $\left\{P_{n}(b)\right\}$ in a neighborhood of 0 . Actually, as in the case of one complex variable, the existence of one derivative in an open domain (e.g., a neighborhood of 0 ) implies holomorphy in the same domain (see $[B]$ ), so we have

Definition $2\left\{P_{n}(b)\right\}$ is uniformly holomorphic in a neighborhood $U$ of $0 \in \mathbf{B}$ (complex Banach Space) if for every $n$, and all $b \in U$ we have

$$
\left\|P_{n}(b)\right\|_{L^{2} \rightarrow L^{2}} \leq c \text { and } P_{n}(b) \text { is Gateaux-differentiable. }
$$

We also have a notion of the «biggest space» in which the variable $b$ can live and maintain the dependence $b \rightarrow P_{n}(b)$ holomorphic. It is given by

Definition 3. $B$ is the space of uniform holomorphy at 0 for $\left\{P_{n}(b)\right\}$ if $\left\{P_{n}(b)\right\}$ is uniformly holomorphic in a neighborhood of 0 and

$$
\sup \left\|\Lambda_{n, 1}(b, \bullet)\right\|_{L^{2} \rightarrow L^{2}}<\infty \text { if and only if } b \in B
$$

We will see later that, for the particular sequence of projection in our problem, proving conditions like (10) or (11) amounts to the proof of some suitable weighted norm inequalities.

\section{Connections with Infinite-Dimensional Toda Flows}

The set-up we have outlined in the previous paragraph is intimately connected with the theory of Toda flows. This theory has been studied 
independently, at first without any reference to orthogonal polynomials (see for example [DLT]).

Let us consider again the "perturbed» space $L^{2}\left(e^{2 t b} d \mu\right)$ where we have added the real parameter $t \in(-\epsilon, \epsilon)$ in the exponential and where we consider the function $b(x)$ fixed.

If we apply the Gram-Schmidt orthogonalization process to the sequence $1, x, x^{2}, \ldots$ embedded in this $L^{2}$ space, we get a sequence $\left\{p_{j, t}(x)\right\}$ of orthogonal polynomials which depends on the parameter $t$. These polynomials satisfy a three-term recurrence

$$
x p_{j, t}(x)=A_{j}(t) p_{j+1, t}(x)+B_{j}(t) p_{j, t}(x)+A_{j-1}(t) p_{j-1, j}(x)
$$

with $j=0,1, \ldots$ and $p_{-1, t}(x)=0$. Since, for each $t$, they are a complete orthonormal system in $L^{2}\left(e^{2 t b} d \mu\right)$, the map $f(x) \rightarrow x f(x)$ induces a bounded linear map $T(t)$ on $l_{+}^{2}$ that can be represented with an infinite tri-diagonal matrix

(2)

$$
T(t)=\left[\begin{array}{ccccc}
B_{0}(t) & A_{0}(t) & 0 & 0 & \ldots \\
A_{0}(t) & B_{1}(t) & A_{1}(t) & 0 & \ldots \\
0 & A_{1}(t) & B_{2}(t) & A_{2}(t) & \ldots \\
0 & 0 & A_{2}(t) & B_{3}(t) & A_{3}(t) \ldots \\
\vdots & \vdots & \vdots & &
\end{array}\right]
$$

If our measure $d \mu$ (and our polynomials) live on the closed interval $[-1,1]$, we have the following explicit formulae for the recursion coefficients (see, e.g., Szegö $[\mathrm{Sz}]$ )

$$
\begin{gathered}
A_{j}(t)=\int_{-1}^{1} x p_{j, t}(x) p_{j+1, j}(x) e^{2 t b(x)} d \mu(x) . \\
B_{j}(t)=\int_{-1}^{1} x p_{j, t}^{2}(x) e^{2 t b(x)} d \mu(x) .
\end{gathered}
$$

We claim that, in general, the operator $T(t)$ is a solution of the (infinite dimensional, non-linear) Toda equation, which can be written as

$$
\dot{T}=[T, \tilde{b}(T)]
$$

where the square brackets stand for the commutator of two operators and $\tilde{b}(T)$ is a sort of Hilbert Transform obtained in the following way: first we use 
operator calculus to define the function $b$ evaluated at $T$, getting some symmetric infinite matrix

$$
b(T)=\left(\begin{array}{ccc} 
& & b_{+} \\
& d & \\
b_{-} & & \backslash
\end{array}\right]
$$

which we have separated in (5) into lower triangular block $\left(b_{-}\right)$, diagonal $(d)$, and upper triangular block $\left(b_{+}\right)$, then we define

$$
\tilde{b}(T)=\left(\begin{array}{ccc} 
& & -b_{-}^{T} \\
& 0 & \\
b_{-} & & \backslash
\end{array}\right]
$$

i.e., we put zeros on the diagonal and replace the upper triangular block $\left(b_{+}\right)$ with the transpose of the lower triangular block $\left(b_{-}\right)$multiplied by -1 .

Proof of the claim

First we want to show that the solution of (4) is expressed by

$$
T(t)=Q^{T}(t) T(0) Q(t)
$$

where the infinite orthogonal matrix $Q(t)$ comes from

$$
e^{t b(T(0))}=Q(t) R(t)
$$

The right hand side of (8) is the $Q R$ decomposition of the 1.h.s.; this decomposition is uniquely determined by asking that $Q$ be orthogonal and $R$ upper triangular (see [DLT]). After showing that (7) is indeed a solution of (9), we will show that the $T(t)$ so obtained coincides with the $T(t)$ in (2).

In the computations that follow, we will assume implicitly that matrices denoted by capital letters are functions of $t$, we will denote differentiation with respect to $t$ with a dot, and use the notation $T_{0}=T(0)$. By differentiating both members of (7) we get

$$
\begin{aligned}
\dot{T} & =\dot{Q}^{T} T_{0} Q+Q^{T} T_{0} \dot{Q} \\
& =\dot{Q}^{T} Q T Q^{T} Q+Q^{T} Q T Q^{T} \dot{Q} \\
& =\dot{Q}^{T} Q T+T Q^{T} \dot{Q} .
\end{aligned}
$$

The second equality is obtained by plugging in the expression for $T(0)$ that comes from (7), the third equality holds just because $Q^{T} Q=I$.

We want to check that

$$
\dot{T}=[T, \tilde{b}(T)]=T \tilde{b}(T)-\tilde{b}(T) T
$$


but, looking at the last term of (9), this amounts to showing that

$$
\tilde{b}(T)=Q^{T} \dot{Q}
$$

Notice that, by the definition of $\tilde{b}(T)$, both terms of this equality must be skew-adjoint, therefore if the equality holds we must have

$$
\left(Q^{T} \dot{Q}\right)^{T}=\dot{Q}^{T} Q=-Q^{T} \dot{Q}
$$

and this is true, as we can see differentiating the identity $Q^{T} Q=I$.

To prove (10) we differentiate (8) and use the fact that the exponential of an operator commutes with that operator. We get

$$
b\left(T_{0}\right) Q R=\dot{Q} R+Q \dot{R}
$$

and multiplying both terms by $Q^{T}$ on the left and by $R^{-1}$ on the right we get

$$
Q^{T} b\left(T_{0}\right) Q=Q^{T} \dot{Q}+\dot{R} R^{-1}
$$

which, after noticing that $Q^{T} b\left(T_{0}\right) Q=b\left(Q^{T} T_{0} Q\right)=b(T)$, can be rewritten as

$$
b(T)=Q^{T} \dot{Q}+\dot{R} R^{-1} .
$$

Let us now go back to the block notation for $b(T)$ that we used in (5), and show that, in that notation, our last equality (12) becomes

$$
\left[\begin{array}{ccc}
\backslash & & b_{+} \\
& d & \\
b_{-} & & \backslash
\end{array}\right]=\left[\begin{array}{ccc} 
& & -b_{-}^{T} \\
& 0 & \\
b_{-} & & \backslash
\end{array}\right]+\left(\begin{array}{ccc}
\backslash & & b_{+}+b_{-}^{T} \\
& d &
\end{array}\right] .
$$

In fact, we know that the first matrix on the right hand side of (12) is skewadjoint, and we know that the second one is upper triangular (because differentiating, inverting, or multiplying triangular matrices we still get triangular matrices). This knowledge induces a chain of deductions that allows us to «fill in» with the proper blocks the two matrices on the right hand side of (13). The diagonal of the first matrix on the right hand side of (13) must be 0 and this forces the diagonal of the second matrix to be $d$. Since this second matrix is upper triangular, we know that the lower triangular block is 0 and this forces the lower triangular block of the first matrix to be $b_{-}$. By skew-adjointness, the upper triangular block of the first matrix is $-b_{-}^{T}$ and this forces the same block in the other matrix to be $b_{+}+b_{-}^{T}$, completing the picture. 
The equality (12) written in the block notation (13) shows in particular that

$$
Q^{T} \dot{Q}=\left[\begin{array}{ccc} 
& & -b_{+}^{T} \\
& 0 &
\end{array}\right]=\tilde{b}(T)
$$

but this is the identity (10) we wanted to prove, so (7) is indeed a solution of (4).

Now we want to check that the solution $T(t)$ of the Toda equation defined by (7) is the same $T(t)$ defined in (2) as the representation in the orthogonal polynomial basis $\left\{P_{j, t}(x)\right\}$ of the operator $M_{x}: f(x) \rightarrow x f(x)$. To do that, notice that we can write

$$
e^{b t} S_{n}(b t) e^{-b t}=e^{b t} R^{-1} R S_{n}(b t) R^{-1} R e^{b t}=Q S_{n}(0) Q^{-1} .
$$

Here, as in the first paragraph, $S_{n}(b t)$ is the partial sum projection operator for the orthogonal polynomials $P_{j, t}(x) \in L^{2}\left(e^{2 b t} d \mu\right)$, while $R^{-1}$ is the upper triangular infinite matrix representing the coefficients of the «perturbed» basis of $\left\{\boldsymbol{P}_{j, t}(x)\right\}$ in terms of the «unperturbed» one $\left\{\boldsymbol{P}_{j, 0}(x)\right\}$, and finally $Q(t)$ is an orthogonal transformation defined by

$$
Q(t)=e^{b t} R^{-1}(t)
$$

Let us point out that the action of $R$ is given by

$$
\sum_{j=0}^{\infty} c_{j} p_{j, 0}(x) \stackrel{R^{\prime \prime}}{\rightarrow} \sum_{j=0}^{\infty} c_{j} p_{j, t}(x) \stackrel{R}{\rightarrow} \sum_{j=0}^{\infty} c_{j} p_{j, 0}(x) .
$$

while the action of $Q$ is given by

$$
\sum_{j=0}^{\infty} c_{j} p_{j, 0}(x) \stackrel{Q}{\rightarrow} \sum_{j=0}^{\infty} c_{j} p_{j, t}(x) e^{b(x) \iota} \stackrel{Q^{-1}}{\rightarrow} \sum_{j=0}^{\infty} c_{j} p_{j, 0}(x) .
$$

These last two diagrams show that $S_{n}(0)=R S_{n}(b t) R^{-1}$, an identity that we have implicitly used in (14). Also they show the orthogonality of $Q$.

We observe now that the matrix of $T(0)$, defined as in (2), represents the multiplication operator $\boldsymbol{M}_{x}$ in the orthonormal basis $\left\{\boldsymbol{P}_{j, 0}\right\}$. We also have

$$
b(T(0))=M_{b(x)}
$$

and 


$$
e^{t b(T(0))}=e^{t M_{b}}=M_{e^{t b}}=e^{t b}
$$

In the last step, we have simply used again the shorthand of indicating a multiplication operator directly by the multiplicator function.

We observe that (15), because of (19), give us the $Q R$ decomposition of $e^{t b(T(0))}$, and therefore the $Q$ appearing in (15) is the same one that appears in the solution of the Toda equation (4). Because of the action of $Q$, we see that the $T(t)$ in (2) is the same as in (7).

\section{The Role of Weighted Norm Inequalities}

According to Definition 2 in Section 2, the sequence of operators $\left\{P_{n}(b)\right\}$ is uniformly holomorphic in a neighborhood $U$ of $0 \in B$ if $P_{n}(b)$ is uniformly bounded in operator norm and Gateaux-differentiable for $b \in U$.

It turns out that, for the particular sequence of projections $P_{n}(b)=e^{b} S_{n}(0) e^{-b}$ which we are considering, the uniform boundedness in $U$ implies also Gateauxdifferentiability. The idea that leads to this simplification is due to Coifman, Rochberg, and Weiss $[\mathrm{CR}]$, and goes as follows:

We can write

$$
\left\{P_{n}(b) f\right\}(x)=\int e^{b(x)-b(y)} D_{n}(x, y) f(y) d \mu(y)
$$

where $D_{n}$ is the Dirichlet Kernel for the partial sums operator $S_{n}(0)$ relative to the orthogonal polynomials on $L^{2}(d \mu)$. Let us fix $b$ and multiply it by a complex parameter $z$. We then have

$$
\left\{\frac{d}{d z} P_{n}(z b) f\right\}(x)=\int(b(x)-b(y)) e^{z(b(x)-b(y))} D_{n}(x, y) f(y) d \mu(y)
$$

Notice then that

(3)

$$
\begin{aligned}
& \frac{1}{2 \pi} \int_{0}^{2 \pi} \exp \left\{e^{i \theta}(b(x)-b(y))\right\} e^{-i \theta} d \theta \\
& \quad=\frac{1}{2 \pi} \sum_{k=0}^{\infty} \int_{0}^{2 \pi} \frac{\left((b(x)-b(y)) e^{i \theta}\right)^{k}}{k !} e^{-i \theta} d \theta \\
& \quad=b(x)-b(y)
\end{aligned}
$$


Therefore we can write

(4) $\left\{\frac{d}{d z} P_{n}(z b) f\right\}=\frac{1}{2 \pi} \iint_{0}^{2 \pi} \exp \left\{\left(z+e^{i \theta}\right)(b(x)-(b(y))\} D_{n}(x, y) f(y) d \mu(y) e^{-i \theta} d \theta\right.$

which, using the notation $P_{n \theta}=P_{n}\left(\left(z+e^{i \theta}\right) b\right)$ can be written as

$$
\left\{\frac{d}{d z} P_{n}(z b) f\right\}(x)=\frac{1}{2 \pi} \int_{0}^{2 \pi}\left\{P_{n \theta} f\right\}(x) e^{-i \theta} d \theta
$$

We are assuming by hypothesis

$$
\|b\|<\delta \text { implies }\left\|P_{n}(b)\right\|_{L^{2} \rightarrow L^{2}} \leq c
$$

Therefore, choosing $|z|<1$ and $\|b\|<\delta / 2$, we have

$$
\left\|\frac{d}{d z} P_{n}(z b)\right\|_{L^{2} \rightarrow L^{2}} \leq \frac{1}{2 \pi} \int_{0}^{2 \pi}\left\|P_{n \theta}\right\|_{L^{2} \rightarrow L^{2}} d \theta \leq c .
$$

So the uniform boundedness expressed by (6) implies locally also uniform analicity.

Notice that (6) can be seen as a weighted norm inequality for $S_{n}(0)$. In fact, the assumption that for $\|b\|<\delta$ one has

$$
\left\|P_{n}(b) f\right\|_{L^{2}(d \mu)} \leq c\|f\|_{L^{2}\left(d_{\mu}\right)} \text { for all } \quad f \in L^{2}(d \mu) .
$$

just by setting $f=e^{b} g$, is seen to be equivalent to

$$
\left\|S_{n}(0) g\right\|_{L^{2}\left(e^{2 b} d \mu\right)} \leq c\|g\|_{L^{2}\left(e^{2 b} d \mu\right)} \text { for all } g \in L^{2}\left(e^{2 b} d \mu\right) .
$$

We will see that in many cases the partial sums operator looks essentially like a Hilbert Transform, while $e^{2 b}$ is a good $A_{p}$ weight when $b$ has a small BMO norm. This allows us to prove weighted norm inequalities like (8) using the results of Hunt, Muckenhaupt, and Wheeden (see [HMW] and also [CF]).

In order to identify BMO also as the space of uniform analyticity at 0 , we need to prove, according to Definition 3 in $\$ 2$, that

$$
b \in \text { BMO if and only if } \sup _{n}\left\|\Lambda_{n 1}(b, \bullet)\right\|_{L^{2} \rightarrow L^{2}}<\infty \text {. }
$$


Let us compute the Gateaux differential that appears in (9). We have

$$
\begin{aligned}
\left.\frac{d}{d t} P_{n}(t b)\right|_{t=0} & =\left.\frac{d}{d t}\left(e^{t b} S_{n}(0) e^{-t b}\right)\right|_{t=0} \\
& =b e^{t b} S_{n}(0) e^{-t b}+\left.e^{t b} S_{n}(0)\left(-b e^{t b}\right)\right|_{t=0} \\
& =\left[b, S_{n}(0)\right]
\end{aligned}
$$

So condition (9) can be rewritten asking that there exist $c_{1}, c_{2}>0$ such that

$$
c_{1}\|b\|_{*} \leq \sup _{n}\left\|\left[b, S_{n}(0)\right]\right\|_{L^{2} \rightarrow L^{2}} \leq c_{2}\|b\|_{*}
$$

where we indicate with a * subscript the BMO norm.

Actually, the inequality on the right is a consequence of uniform holomorphy (which implies the boundedness of all derivatives), so we only need to prove the one on the left.

\section{Perturbation Theory of a Family of Orthogonal Polynomials Introduced by Szegö}

In Szegö's classic book [Sz] on orthogonal polynomials, the starting point for the proof of his equiconvergence results is the study of a particular family of orthogonal polynomials in $L^{2}(\mathbf{T}, d \mu)$. The measure $d \mu$ on the unit circle is given by

$$
d \mu(\theta)=\frac{1}{g(\theta)} \frac{d \theta}{2 \pi}
$$

with $g$ trigonometric polynomial of degree $m$ and $g(\theta)>0$ for all $\theta \in[0,2 \pi]$. It turns out that for any fixed $g$, even though it is not easy to find a closed formula for the first $m$ orthogonal polynomials, there is in fact a simple expression for them when the degree is greater than or equal to $m$. This restriction does not affect Szegö's proofs, and will not affect ours either.

We have the following:

Proposition 1 The sequence $\left\{\phi_{j}(z)\right\}$ of complex polynomials (normalized with a strictly positive coefficient for $z^{j}$ ) satisfying

$$
\frac{1}{2 \pi} \int_{-\pi}^{\pi} \phi_{j}(z) \overline{\phi_{k}(z)} \frac{1}{g(\theta)} d \theta=\delta_{j k}
$$

( $z=e^{i \theta}$ in the integral) is given, when $j=m, m+1, \ldots$, by 


$$
\phi_{j}(z)=z^{j-m} h^{*}(z)
$$

where $h(z)$ is a complex polynomial of degree $m$ such that

$$
g(\theta)=|h(z)|^{2} \quad \text { when } z=e^{i \theta}
$$

and $h^{*}$ is the reciprocal polynomial of $h$, i.e.,

$$
h^{*}(z)=z^{m} \bar{h}\left(\frac{1}{z}\right)
$$

PROOF. The existence of representation (4) for the positive polynomial $g$ is a special case of a more general one, from $H^{p}$ theory, that holds in fact for all $f$ such that $\log f \in L^{1}(\mathbf{T})$. Notice that we can choose $h(0)>0$ and $h(z)$ $\neq 0$ for $|z|<1$.

We can rewrite (3) as

$$
\phi_{j}(z)=z^{j} \bar{h}\left(z^{-1}\right) .
$$

Here, as before in (5), we use the convention that if $h(z)$ is a polynomial in $z, \bar{h}$ is the polynomial obtained from $h$ by conjugating the coefficients (not $z$ ).

Plugging (6) into (2) we can easily verify the case $j=k$ just remembering that on the unit circle we have $\bar{z}=z^{-1}$.

To prove the case $j \neq k$ we actually show that

$$
\frac{1}{2 \pi} \int_{-\pi}^{\pi} \phi_{j}(z) \overline{\varrho(z)} \frac{1}{g(\theta)} d \theta=0 \quad \text { when } z=e^{i \theta}
$$

for any polynomial $\varrho$ of degree $j-1$. In fact we get

$$
\begin{aligned}
\frac{1}{2 \pi i} \int_{\Gamma} z^{j} \bar{h}\left(z^{-1}\right) \frac{\bar{\varrho}\left(z^{-1}\right)}{h(z) \bar{h}\left(z^{-1}\right)} \frac{d z}{z} & =\frac{1}{2 \pi i} \int_{\Gamma} \frac{z^{j-1} \bar{\varrho}\left(z^{-1}\right)}{h(z)} d z \\
& =\frac{1}{2 \pi i} \int_{\Gamma} \frac{\varrho^{*}(z)}{h(z)} d z=0 .
\end{aligned}
$$

The last equality holds because we are integrating along the unit circle $\Gamma$ a meromorphic function with no poles inside. 
Given a closed expression of the polynomials, one can give an expression of the Dirichlet kernel of the partial sums operator (we refer to $[\mathrm{Sz}]$, p. 292, for the algebraic details). In our case we have

$$
D_{n}(\theta, \varphi)=\frac{h\left(e^{i \theta}\right) \overline{h\left(e^{i \psi}\right)}-e^{i(n+1)(\theta-\psi)} \overline{h\left(e^{i \theta}\right)} h\left(e^{i \psi}\right)}{1-e^{i(\theta-\psi)}} .
$$

This expression is valid whenever $n \geq m$, and shows explicitly the dependence of the kernel on the weight. Following our general plan we look at $P_{n}(b)$ which is now given by

$$
\text { (9) } \begin{aligned}
\left\{P_{n}(b) f\right\}(\theta) & =\frac{1}{2 \pi} \int_{-\pi}^{\pi} e^{b(\theta)} D_{n}(\theta, \psi) e^{-b(\psi)} \frac{f(\psi)}{g(\psi)} d \psi \\
& =\frac{1}{2 \pi} \int_{-\pi 1}^{\pi} e^{b(\theta)-b(\psi)} \frac{f(\psi)}{1-e^{i(\theta-\psi)}}\left\{\frac{h\left(e^{i \theta}\right)}{h\left(e^{i \psi}\right)}-e^{i(n+1)(\theta-\psi)} \frac{\overline{h\left(e^{i \theta}\right)}}{\overline{h\left(e^{i \psi}\right)}}\right\} d \psi \\
& =\left\{\left(P_{n}^{\prime}(b)-P_{n}^{\prime \prime}(b)\right) f\right\}(\theta)
\end{aligned}
$$

where

$$
\left\{\left(P_{n}^{\prime}(b) f\right\}(\theta)=\frac{1}{2 \pi} \int_{-\pi}^{\pi} e^{b(\theta)} h\left(e^{i \theta}\right) \frac{f(\psi)}{1-e^{i(\theta-\psi)}} e^{-b(\psi)} h\left(e^{i \psi}\right)^{-1} d \psi\right.
$$

and

$\left(10^{\prime \prime}\right)\left\{\left(P_{n}^{\prime \prime}(b) f\right\}(\theta)=\frac{1}{2 \pi} \int_{-\pi}^{\pi} e^{i(n+1) \theta+b(\theta)} \overline{h\left(e^{i \theta}\right)} \frac{f(\psi)}{1-e^{i(\theta-\psi)}} e^{-i(n+1) \psi-b(\psi)} \overline{h\left(e^{i \psi}\right)^{-1}} d \psi\right.$.

The operators in $\left(10^{\prime}\right)$ and $\left(10^{\prime \prime}\right)$ consist of a singular integral (essentially a Hilbert transform) conjugated by a multiplication operator on the left and the inverse of the same multiplication on the right. Let us look at a third operator

$\left(10^{\prime \prime \prime}\right)\left\{\left(\mathrm{P}_{n}^{\prime \prime \prime}(b) f\right\}(\theta)=\frac{1}{2 \pi} \int_{-\pi}^{\pi} e^{\mathrm{Reb}(\theta)} g(\theta)^{1 / 2} \frac{f(\psi)}{1-e^{i(\theta-\psi)}} e^{-\mathrm{Reb}(\psi)} g(\psi)^{-1 / 2} d \psi\right.$

which, by the result in [HMW], is bounded for $e^{\operatorname{Reb}(\theta)} g(\theta)^{1 / 2} \in A_{2}$. This last condition is satisfied when the real part of $b(\theta)$ has BMO-norm smaller 
than some suitable $\delta$. (See [GCRF], chapter 4, and notice that $g(\theta)$ is a polynomial without zeroes for every $\theta$ ).

The same condition on $b(\theta)$ implies uniform boundedness also for $\left(10^{\prime}\right)$ and $\left(10^{\prime \prime}\right)$, just noticing that they are both obtained from (10"') multiplying on the left by a complex function of modulus 1 and on the right by the inverse of that same function. By what we have seen in the previous paragraph, for this particular kind of operators uniform boundedness implies uniform holomorphy, so the family of projections $\left\{\boldsymbol{P}_{n}(b)\right\}$ is uniformly holomorphic for $b$ in a neighborhood of $0 \in \mathrm{BMO}$.

By Kerzman-Stein formula (2.5), or also using the Toda Flow identities of Section 3, the same uniform result holds for the self adjoint projections $V_{n}(b)$.

The next step of the plan consists in showing that BMO is in fact the space of uniform holomorphy of the $\left\{P_{n}(b)\right\}$, and, as we have seen in general, that amounts to proving the inequality

$$
c_{1}\|b\|_{\|} \leq \sup _{n}\left\|\left[b, S_{n}(0)\right]\right\|_{L^{2} \rightarrow L^{2}} .
$$

To do that we define

$$
f_{\theta}(\psi)=\chi_{I}(\psi)\left(1-e^{i(\theta-\psi)}\right) h\left(e^{i \psi}\right)
$$

where $I$ is a closed subinterval of $[0,2 \pi]$.

We have

(13) $\left\{\left[b, S_{n}(0)\right] f_{\theta}\right\}(\theta)=\frac{1}{2 \pi} \int_{I}(b(\theta)-b(\psi))\left\{h\left(e^{i \theta}\right)-e^{i(n+1)(\theta-\psi)} \frac{h\left(e^{i \psi}\right) \overline{h\left(e^{i \theta}\right)}}{\overline{h\left(e^{i \psi}\right)}}\right\} \mathrm{d} \psi$.

By Riemann-Lebesgue lemma we have

(14) $\lim _{n \rightarrow \infty}\left\{\left[b, S_{n}(0)\right] f_{\theta}\right\}(\theta)=\frac{1}{2 \pi} \int_{I}(b(\theta)-b(\psi)) h\left(e^{i \theta}\right) d \psi=\frac{|I|}{2 \pi} h\left(e^{i \theta}\right)\left(b(\theta)-m_{I}(b)\right)$

where $m_{I}(b)$ is the average of the function $b$ over $I$. By Fatou's lemma we have

$$
|I| \int_{I}\left|b(\theta)-m_{I}(b)\right| d \theta \leq c \limsup _{n \rightarrow \infty} \int_{I}\left|\left\{\left[b, S_{n}(0)\right] f_{\theta}\right\}(\theta)\right| \frac{d \theta}{\left|h\left(e^{i \theta}\right)\right|}
$$

Therefore (11) will hold if we can bound the right hand side of this by 


$$
c|I|^{2} \sup _{n}\left\|\left[b, S_{n}(0)\right]\right\|
$$

where $c$ does not depend on $n$ and $I$.

Let $\alpha$ be the midpoint of $I$; a straightforward computation allows us to rewrite (12) in the form

(16) $f_{\theta}(\psi)=e^{i(\theta-\alpha) / 2} \sin \frac{\theta-\alpha}{2} f_{1}(\psi)+e^{i(\theta-\alpha) / 2} \cos \frac{\theta-\alpha}{2} f_{2}(\psi)$

where $f_{1}$ and $f_{2}$ do not depend on $\theta$, and are given by

$$
\begin{aligned}
& f_{1}(\psi)=-2 i \chi_{I}(\psi) h\left(e^{i \psi}\right) e^{i(\alpha-\psi) / 2} \cos \frac{\alpha-\psi}{2} . \\
& f_{2}(\psi)=-2 i \chi_{I}(\psi) h\left(e^{i \psi}\right) e^{i(\alpha-\psi) / 2} \sin \frac{\alpha-\psi}{2} .
\end{aligned}
$$

Using (16) and Schwarz's inequality, we get

(17)

$$
\begin{aligned}
& \int_{I}\left|\left\{\left[b, S_{n}(0)\right] f_{\theta}\right\}(\theta)\right| \frac{d \theta}{\left|h\left(e^{i \theta}\right)\right|} \\
& \leq\left(\int_{I}\left|\sin \frac{\theta-\alpha}{2}\right|^{2} d \theta\right)^{1 / 2}\left(\int_{I}\left|\left\{\left[b, S_{n}(0)\right] f_{1}\right\}(\theta)\right|^{2} \frac{d \theta}{\left|h\left(e^{i \theta}\right)\right|^{2}}\right)^{1 / 2} \\
& \quad+\left(\int_{I}\left|\cos \frac{\theta-\alpha}{2}\right|^{2} d \theta\right)^{1 / 2}\left(\int_{I}\left|\left\{\left[b, S_{n}(0)\right] f_{2}\right\}(\theta)\right|^{2} \frac{d \theta}{\left|h\left(e^{i \theta}\right)\right|^{2}}\right)^{1 / 2} .
\end{aligned}
$$

This last inequality holds for every $n$, so we can take the upper limit of the left hand side. Also, we notice that the right factors in the two terms on the right hand side are, for $k=1,2$, exactly the $L^{2}(d \mu)$ norms of $\left[b, S_{n}(0)\right] f_{k}$, since $d \mu(\theta)=\left|h\left(e^{i \theta}\right)\right|^{-2} d \theta$.

We have

(18) $\quad \limsup _{n \rightarrow \infty} \int\left|\left\{\left[b, S_{n}(0)\right] f_{\theta}\right\}(\theta)\right| \frac{d \theta}{\left|h\left(e^{i \theta}\right)\right|} \leq c_{1}|I|^{3 / 2} A\left\|f_{1}\right\|_{L^{2}(d \mu)}$

$$
+c_{2}|I|^{1 / 2} A\left\|f_{2}\right\|_{L^{2}\left(d_{\mu}\right)}
$$

where $c_{1}, c_{2}$ de not depend on $n$ or $I$ and 


$$
A=\sup \left\|\left[b, S_{n}(0)\right]\right\|_{L^{2}(d \mu) \rightarrow L^{2}(d \mu)} \text {. }
$$

To complete our proof we only need to check that

$$
\left\|f_{1}\right\|_{L^{2}(d \mu)} \leq c_{3}|I|^{1 / 2} \text { and }\left\|f_{2}\right\|_{L^{2}(d \mu)} \leq c_{4}|I|^{3 / 2}
$$

and this is easily seen to be true by our choice of $f_{1}$ and $f_{2}$.

\section{Generalizations and the Role of VMO}

In the previous paragraph we have proven that the family of orthogonal projections

$$
V_{n}(b)=e^{b} S_{n}(b) e^{-b}: L^{2}(d \mu) \rightarrow L^{2}(d \mu)
$$

is uniformly holomorphic (Definition 2.2) for $b$ belonging to a neighborhood of 0 in BMO. Also we have shown that BMO is the space of uniform holomorphy at 0 (Definition 2.3). Notice that our $d \mu$ is a measure on $\mathbf{T}$ expressed via a non-vanishing weight of the form (5.1).

In fact, as long as we satisfy the proper estimates, we could have started from a measure $d \mu$, given by a weight with zeroes. The intuitive idea is that a BMO function can contain unbounded logarithmic spikes (and the exponential of a negative spike can be 0 ); here we want to make this idea more precise, getting an analyticity result that holds for a «starting set» of orthogonal polynomials more general than those of the form (5.2).

We need first to introduce an important closed Banach subspace of BMO It is called VMO (Vanishing Mean Oscillation) and is defined by

$$
\mathrm{VMO}=\left\{f \in \mathrm{BMO}: \lim _{a \rightarrow 0} M_{a}(f)=0\right\}
$$

where

$$
M_{a}(f)=\sup _{|I| \leq a} \frac{1}{|I|} \int_{I}\left|f(x)-f_{I}\right| d x
$$

As usual $I$ denotes an interval and $f_{I}$ the average of $f$ on $I$. Notice that, with this notation, the usual BMO norm is given by

$$
\|f\|_{*}=\lim _{a \rightarrow \infty} M_{a}(f)
$$


This space has been studied by Sarason, and in [Sa] one can find the statement and proof of some basic properties; we are going to use, in particular, the fact that VMO is the closure, in BMO-norm, of the subspace of continuous functions.

Lemma 1 Given a real-valued function $\gamma \in$ V.M.O. (T) one can find a sequence of strictly positive trigonometric polynomials $g_{j}(\theta)$ such that

$$
\lim _{j \rightarrow \infty}\left\|\gamma(\theta)-\log \left(\frac{1}{g_{j}(\theta)}\right)\right\|_{*}=0
$$

Proof. Continous functions are BMO-dense in VMO and since $\|f\|_{*} \leq 2\|f\|_{\infty}$ we only need to show that for any real-valued $\beta(\theta) \in C(\mathbf{T})$ one can find a sequence of strictly positive trigonometric polynomials $g_{j}(\theta)$ such that

$$
\lim _{j \rightarrow \infty}\left\|\beta(\theta)-\log \left(\frac{1}{g_{j}(\theta)}\right)\right\|_{\infty}=0
$$

we have

(5) $\left|\beta(\theta)-\log \left(\frac{1}{g_{j}(\theta)}\right)\right|=\left|\log \left(g_{j}(\theta) e^{\beta(\theta)}\right)\right|=\left|\log \left(1+e^{\beta(\theta)}\left(g_{j}(\theta)-e^{-\beta(\theta)}\right)\right)\right|$.

And the compactness of $\mathbf{T}$ implies the existence of two positive constants $c_{1}, c_{2}$ such that

$$
c_{1} \leq e^{\beta(\theta)} \leq c_{2}
$$

But this implies that the quantity in (5) can be made uniformly small just by uniform approximation of the positive continuous function $e^{-\beta(\theta)}$ with the positive polynomials $g_{j}(\theta)$.

Lemma 2 The radius of analycity $\delta$ for the mapping relative to the orthogonal polynomials (5.2) does not depend on the particular $g(\theta)$ contained in the weight. 
PROOF. Going back to the previous paragraph, we notice that we have analyticity for those functions $b \in \mathrm{BMO}$ wich satisfy

$$
e^{\operatorname{Re} b(\theta)} \sqrt{g(\theta)} \in A_{2} .
$$

It is a property of $A_{2}$ weights to be invariant under multiplication by functions bounded away from 0 and infinity (see [GCRF], chapter 4). On the other hand the first factor in (7) is an $A_{2}$ weight in general only if $\|\operatorname{Re} b\|_{*}<\delta$.

Remark. If one makes additional assumptions on the nature of $b$, one can find functions with large BMO norm whose exponential is still in $A_{2}$. This surprising fact is well illustrated by a theorem of Garnett and Jones which says the following

$$
A(b)=\sup \left\{\lambda>0: e^{\lambda b} \in A_{2}\right\} \approx\left(\underset{\mathrm{BMO}}{\operatorname{dist}}\left\{b, L^{\infty}\right\}\right)^{-1},
$$

where the symbol « $\approx$ » means «same order of magnitude» and where

$$
\underset{\mathrm{BMO}}{\operatorname{dist}}\left\{b, L^{\infty}\right\}=\inf \left\{\|b-f\|_{*}: f \in L^{\infty}\right\} .
$$

In particular, the exponential of any VMO function (regardless of the BMO norm) is an $A_{2}$ weight.

We can now state our general results as follows

Theorem 1. The uniform analicity of the mapping $b \rightarrow V_{n}(b)$ holds for all orthogonal polynomials on $L^{2}(d \mu)$ where $d \mu$ is a measure on $\mathbf{T}$ of the form

$$
d \mu(\theta)=e^{2(\gamma(\theta)+\beta(\theta))} \frac{d \theta}{2 \pi}
$$

with $\gamma(\theta)$ any real-valued VMO function and $\beta(\theta)$ a complex-valued BMO function such that

$$
\|\operatorname{Re} \beta\|_{*}<\delta
$$

where $\delta$ is the radius of analyticity for the Szegö polynomials (5.2).

The new radius of analyticity, if we start from the polynomials relative to the measure (10), is given by

$$
\delta^{\prime}=\delta-\|\operatorname{Re} \beta\|_{*}
$$


Proof. Let us fix some BMO function $\beta$ satisfying (11). We know that starting from a measure of the form (5.1) we can expand $V_{n}(b) f$ into a series of multilinear operators (2.8) satisfying an estimate (2.9). Let us define a new «starting space» for our perturbation

$$
L^{2}(d \mu)=L^{2}\left(e^{2 \beta(\theta)} \frac{1}{g(\theta)} \frac{d \theta}{2 \pi}\right)
$$

Then let us denote, according to the notations of Section 2, by $S_{n}$ and $V_{n}$ the projections relative to the $L^{2}$ space in (13), while we denote the same projections relative to the $\beta=0$ situation by $S_{n}^{0}$ and $V_{n}^{0}$. We have

$$
\begin{aligned}
& S_{n}(0)=S_{n}^{0}(\beta) \\
& S_{n}(b)=S_{n}^{0}(b+\beta) \\
& V_{n}(b)=e^{b} S_{n}^{0}(b+\beta) e^{-b}
\end{aligned}
$$

where the operators in the «new» space are expressed in terms of those in the «old» space. Using these identities we can write

$$
\left\|V_{n}(b)\right\|_{L^{2}(d \mu) \rightarrow L^{2}(d \mu)}=\left\|e^{b+\beta} S_{n}^{0}(b+\beta) e^{-(b+\beta)}\right\|_{L^{2}(g) \rightarrow L^{2}(g)}
$$

where we denote by $L^{2}(g)$ the space relative to the measure $-\frac{1}{g(\theta)} \frac{d \theta}{2 \pi}$.

By the results of Section 5, we have uniform holomorphy for $V_{n}(b)$ if

$$
\|\operatorname{Re}(b+\beta)\|_{*}<\delta
$$

and this holds for any $b$ whose real part has BMO norm less than the $\delta^{\prime}$ in (12).

Finally, lemma 1 and lemma 2 imply that we can substitute $1 / g(\theta)$ in the right hand side of (13) with $e^{2 \gamma(\theta)}$ where $\gamma \in \mathrm{VMO}$. We remark that weights of the form (10) can have any number of zeros (only with restrictions on the rate of decay of the weight around each zero).

\section{The Operation on Infinite Matrices Expressed Via the Hilbert Transform and a Remarkable Bilinear Operator}

Starting from our knowledge of the operator-valued function

$$
b \rightarrow V_{n}(b)
$$


and given the identities (3.14-3.15), i.e.,

$$
V_{n}(b)=e^{b} S_{n}(b) e^{-b}=Q(b) S_{n}(0) Q(b)^{-1}
$$

where

$$
Q(b)=e^{b} R^{-1}(b)
$$

is an orthogonal transformation depending on $b \in \mathrm{BMO}$, it is a natural problem to study the mapping

$$
b \rightarrow Q(b)
$$

The first step of this study is to compute and understand the Gateaux differential of (1) at 0 . We claim that

$$
\left.\frac{d}{d t} Q(b t)\right|_{t=0}=\tilde{M}_{b}
$$

where $M_{b}$ is the operator of multiplication by $b$ and $\tilde{M}_{b}$ is defined as in (3.5-3.6).

In fact, by the Kerzman-Stein formula (2.5) we have

$$
V_{n}(b)=P_{n}(b)\left(I+P_{n}(b)-P_{n}(-b)\right)^{-1}
$$

therefore

$$
\text { (3) } \begin{aligned}
\left.\frac{d}{d t} V_{n}(b t)\right|_{t=0} & =\left.\frac{d}{d t} P_{n}(t b)\right|_{t=0}-\left.P_{n}(0) \frac{d}{d t}\left\{P_{n}(t b)-P_{n}(-t b)\right\}\right|_{t=0} \\
& =\left[b, S_{n}(0)\right]-S_{n}(0)\left\{\left[b, S_{n}(0)\right]+\left[b, S_{n}(0)\right]\right\} \\
& =\left\{I-2 S_{n}(0)\right\}\left[b, S_{n}(0)\right] \\
& =\left[\tilde{M}_{b}, S_{n}(0)\right] .
\end{aligned}
$$

The last equality can be checked by writing the operators as infinite matrices, remembering that $S_{n}(0)$ is the identity on the first $(n+1) \times(n+1)$ entries while

$$
b=M_{b}=\left(m_{n k}\right) \text { implies } \quad \tilde{M}_{b}=\left(\operatorname{sgn}(n-k) m_{n k}\right)
$$

Because of identity (3.14), we have

$$
\left[\dot{Q}(0), S_{n}(0)\right]=\left[\tilde{M}_{b}, S_{n}(0)\right]
$$


and since this commutator equality holds for $n=0,1,2, \ldots$, and $\dot{Q}^{*}=-\dot{Q}$, we can conclude that

$$
\dot{Q}(0)=\tilde{M}_{b}
$$

While studying the explicit form that the operator $\tilde{M}_{b}$ takes for some explicit sets of orthogonal polynomials, we discovered a remarkable formula. It is an operator identity expressing the operation $\sim$ on infinite matrices via Hilbert Transforms on the circle, and via the bilinear operator

$$
B(b, f)(x)=\frac{1}{2 \pi} \int b(x-\theta) f(x-2 \theta) \cot \frac{\theta}{2} d \theta
$$

Notice that it is still an open problem to establish whether operators like (6), or their analogues on the real line, are $L^{2}$-bounded for $b \in L^{\infty}$ (or $b \in \mathrm{BMO}$ ).

The context in which the formula arises is cosine polynomials (Chebychev polynomials after a change of variable).

Consider the $L^{2}$ space of functions of the form

$$
f(x)=\sum_{j=0}^{\infty} f_{j} \cos j x \quad\left(\mathrm{~b}_{j} \in \mathbf{C}\right)
$$

and the multiplication operator

$$
\left\{M_{b} f\right\}(x)=b(x) f(x)
$$

where

$$
b(x)=\sum_{j=0}^{\infty} b_{j} \cos j x \quad\left(b_{j} \in \mathbf{C}\right) .
$$

The operator $M_{b}$ can be represented in the basis $\{\cos j x\}$ by an infinite matrix

$$
M_{b}=\left(m_{n k}\right) \quad n, k=0,1,2, \ldots
$$

and we can define

$$
\tilde{M}_{b}=\left(-i \operatorname{sgn}(n-k) m_{n k}\right)
$$

which is the usual definition, apart from the constant factor $-i$. 
Introducing a new variable $\theta$, we can write an integral expression for $\bar{M}_{b}$, in fact

$$
\tilde{M}_{b}=\frac{1}{2 \pi} \cdot \int_{1}^{2 \pi} U_{\theta} M_{b} U_{-\theta} \cot \frac{\theta}{2} d \theta
$$

where

$$
\begin{gathered}
U_{\theta} f=\sum_{j=0}^{\infty} e^{-i j \theta} f_{j} \cos j x, \\
U_{-\theta} f=\sum_{j=0}^{\infty} e^{i j \theta} f_{j} \cos j x .
\end{gathered}
$$

Now, we have

(14) $U_{-\theta} f=\sum_{j=0}^{\infty} f_{j}(\cos j \theta \cos j x+i \sin j \theta \cos j x)$

$$
\begin{aligned}
& =\sum_{j=0}^{\infty} f_{j}\left(\frac{\cos j(x+\theta)+\cos j(\mathrm{x}-\theta)}{2}+\frac{\sin j(x+\theta)-\sin j(x-\theta)}{2}\right) \\
& =\frac{1}{2}\left\{T_{-\theta} f+T_{\theta} f+i\left(T_{-\theta} H f-T_{\theta} H f\right)\right\}
\end{aligned}
$$

where

$$
\left\{T_{\theta} f\right\}(x)=f(x-\theta)
$$

and $H f$ is the Hilbert Transform of $f$ (on the circle).

A similar computation shows that

$$
U_{\theta} f=\frac{1}{2}\left\{T_{-\theta} f+T_{\theta} f-i\left(T_{-\theta} H f-T_{\theta} H f\right)\right\} .
$$

Using (14) and (16), let us compute the operator $U_{\theta} M_{b} U_{-\theta}$. In what follows, in order to make the notation less cumbersome, we will simply write $M$ for $M_{b}$. We have 
(17) $4 U_{\theta} M U_{-\theta}=$

$$
\begin{aligned}
= & U_{\theta}\left\{M T_{-\theta}+M T_{\theta}+i\left(M T_{-\theta} H-M T_{\theta} H\right)\right\} \\
= & T_{-\theta} M T_{-\theta}+\mathrm{T}_{-\theta} M T_{\theta}+i T_{-\theta} M T_{-\theta} H-i T_{-\theta} \mathrm{MT}_{\theta} H \\
& +\mathrm{T}_{\theta} M T_{-\theta}+\mathrm{T}_{\theta} \mathrm{MT}_{\theta}+i \mathrm{~T}_{-\theta} M T_{-\theta} H-i T_{\theta} M T_{\theta} H \\
& -i T_{-\theta} H M T_{-\theta}-i T_{-\theta} H M T_{\theta}+T_{-\theta} H M T_{-\theta} H-T_{-\theta} H M T_{\theta} H \\
& +i T_{\theta} H M T_{-\theta}+i T_{\theta} H M T_{\theta}-T_{\theta} H M T_{-\theta} H+T_{\theta} H M T_{\theta} H .
\end{aligned}
$$

If we plug these sixteen terms back into (12), we notice that the eight terms giving the real part cancel after integration against $\cot \frac{\theta}{2} d \theta$. In fact, we have

$$
\begin{aligned}
\left\{\left(T_{-\theta} M T_{-\theta}+\right.\right. & \left.\left.T_{-\theta} M T_{\theta}+T_{\theta} M T_{-\theta}+T_{\theta} M T_{\theta}\right) f\right\}(x)= \\
= & b(x+\theta) f(x+2 \theta)+b(x+\theta) f(x) \\
& +b(x-\theta) f(x)+b(x-\theta)(x-2 \theta),
\end{aligned}
$$

and these four terms define an even function of $\theta$, which in the integral is multiplied by the odd function $\cot \theta / 2$.

Similarly, the other four real terms applied to $f$ give (using the notation $H f=\tilde{f})$

(19) $\quad H(b(x+\theta) \tilde{f}(x+2 \theta)-b(x+\theta) \tilde{f}(x)-b(x-\theta) \tilde{f}(x)+b(x-\theta) \tilde{f}(x-2 \theta))$

and these cancel, too, if we assume that the integration against $\cot \frac{\theta}{2} d \theta$ can be done before the Hilbert Transform $H$ in the $x$ variable.

Let us now look at the other eight terms in (17); they are

$$
\begin{aligned}
i\left(T_{-\theta} M T_{-\theta} H\right. & -T_{-\theta} M T_{\theta} H+T_{\theta} M T_{-\theta} H-T_{\theta} M T_{\theta} H \\
& \left.-T_{-\theta} H M T_{-\theta}-T_{-\theta} H M T_{\theta}+T_{\theta} H M T_{-\theta}+T_{\theta} H M T_{\theta}\right)
\end{aligned}
$$

and applying them to $f(x)$, we get

(21) $4 \operatorname{Im}\left\{\left(U_{\theta} M U_{-\theta}\right) f\right\}(x)$

$$
\begin{aligned}
= & b(x+\theta) \tilde{f}(x+2 \theta)-b(x+\theta) \tilde{f}(x)+b(x-\theta) \tilde{f}(x)-b(x-\theta) \tilde{f}(x-2 \theta) \\
& +H(-b(x+\theta) f(x+2 \theta)-b(x+\theta) f(x) \\
& +b(x-\theta) f(x)+b(x-\theta) f(x-2 \theta)\} .
\end{aligned}
$$

Plugging this expression into (12), and making the change of variable $\theta \rightarrow-\theta$ in the four integrals that contain $x+\theta$ or $x+2 \theta$, we get 
(22) $\tilde{M} f=\frac{i}{4}\left\{2 \tilde{f}(x) \int b(x-\theta) d m(\theta)\right.$

$$
\begin{aligned}
& -2 \int b(x-\theta) \tilde{\mathrm{f}}(x-2 \theta) d m(\theta) \\
& +\left(2 f(x) \int b(x-\theta) d m(\theta)\right)^{\sim} \\
& \left.+\left(2 \int b(x-\theta) f(x-2 \theta) d m(\theta)\right)^{\sim}\right\}
\end{aligned}
$$

where we have used the notation

$$
d m(\theta)=\cot \frac{\theta}{2} \frac{d \theta}{2 \pi}
$$

and $\operatorname{dm}(\theta)$ lives on $[0,2 \pi]$.

The final formula is

$$
\text { (24) } \begin{aligned}
\{\tilde{M} f\}(x)=\frac{i}{2}\left\{\tilde{f}(x) \tilde{b}(x)-\frac{1}{2 \pi} \int_{0}^{2 \pi} b(x-\theta) \tilde{f}(x-2 \theta) \cot \frac{\theta}{2} d \theta\right. \\
\\
+(f \tilde{b})^{-}(x) \\
\left.+\frac{1}{2 \pi}\left(\int_{0}^{2 \pi} b(x-\theta) f(x-2 \theta) \cot \frac{\theta}{2} d \theta\right)^{\sim}\right\} .
\end{aligned}
$$

\section{References}

[AR $\quad$ B. Alpert and V. Rokhlin. A Fast Algorithm for the Evaluation of Legendre Expansions, Research Report RR-671, Yale Dept. of Computer Science, 1989.

[B $\quad$ M. S. Berger. Nonlinearity and Functional Analysis: Lectures on Nonlinear Problems in Mathematical Analysis, Academic Press, New York, 1977.

[CF $\mid$ R. R. Coifman and C. Fefferman. Weighted Norm Inequalities for Maximal Functions and Singular Integrals, Studia Mathematica 51 (1974), 241-250.

[CM] R. R. Coifman and M. A. M. Murray. Uniform Analyticity of Orthogonal Projections, Trans. Amer. Math. Soc., 312 (1989), 779-817.

[CR $\quad$ R. R. Coifman and R. Rochberg. Projections in Weighted Space, Skew Projections and Inversion of Toeplitz Operators, Integral Equations and Operator Theory, 5 (1982), 142-159. 
[DLT] P. Deift, L. C. Li, and C. Tomei. Toda Flows with Infinitely Many

- Variables, J. Functional Anal. 64 (1985), 358-402.

[G] J. B. Garnett. Bounded Analytic Functions, Academic Press, New York, 1981.

[GCRF] J. García-Cuerva and J. L. Rubio de Francia. Weighted Norm Inequalities and Related Topics, North Holland Mathematics Studies 116, North Holland, Amsterdam, 1985.

[HMW] R. Hunt, B. Muckenhoupt, and R. Wheeden. Weighted Norm Inequalities for the Conjugate Function and Hilbert Transform, Trans. Amer. Math. Soc. 176 (1973), 227-251.

|KS $\quad$ N. Kerzman and E. M. Stein. The Szegö Kernel in Terms of CauchyFantappié Karnels, Duke Math. J. 45 (1978), 197-224.

[R] W. Rudin. Functional Analysis, Mc Graw Hill, 1973.

[Sa $\mid$ D. Sarason. Functions of Vanishing Mean Obscillation, Trans. Amer. Math. Soc., 207 (1975), 391-405.

[Sz] G. Szegö. Orthogonal Polynomials, Fourth Edition, American Mathematical Society, Providence, 1975.

Recibido: 8 de febrero de 1991

\author{
Enrico Laeng \\ Politecnico de Milano \\ Dipartimento di Matematica \\ Piazza Leonardo da Vinci 32 \\ 20133 Milano \\ Italy
}

